Aceptado: Junio 2018

\title{
Las actividades dirigidas en centros deportivos y su valor como elemento de fidelización
}

\section{Group exercise training in sport centers and its value as a retention element}

\author{
Marta Pérez-Villalba ${ }^{1}$, Jerónimo García-Fernández², Ramón Gómez-Chacón ${ }^{3}$ y Nicolás Fernández-Martínez ${ }^{3}$ \\ 1 Centro Universitario San Isidoro, Sevilla (España). \\ 2 Departamento de Educación Física y Deporte, Universidad de Sevilla. (España). \\ 3 Fundación San Pablo Andalucia CEU, Sevilla. (España).
}

Resumen: El objetivo de este estudio fue determinar la demanda de actividades dirigidas (AADD) por parte de las personas abonadas a centros deportivos españoles y determinar si las AADD se presentan como un elemento de fidelización de la instalación. Un total de 15.778 clientes de 97 centros deportivos de ciudades de más de 100.000 habitantes fueron encuestados a través de un cuestionario diseńado ad hoc. Lo resultados muestran una mayor demanda de AADD entre el colectivo de mujeres $(77,5 \%)$ frente a los hombres (45,9\%), y entre las franjas de edad comprendidas entre los 25 y 64 años superando el $60 \%$. Los datos obtenidos confirman que las AADD funcionan como un elemento de fidelización entre el colectivo de personas que hacen $\mathrm{AADD}$ de manera regular, presentandouna mayor permanencia, y un mayor volumen de días y de horas semanales de asistencia.

Palabras clave: Centro fitness, clientes, actividades dirigidas, fidelización.
Abstract: The aim of the study was to determine the demand of group exercise training (GET) by members of Spanish sports centers, and determine if GET could be considered as a retention element. A total of 15,778 members from 97 sports centers in cities with more than 100,000 inhabitants were surveyed through an ad hoc questionnaire. The results show a higher demand for GET among the group of women (77.5\%) than men (45.9\%), and between ages 25 and 64 exceeding 60\%. The data obtained confirm that GET work as a loyalty element among the group of people who attend GET on a regular basis, presenting a greater membership tenure, and a greater volume of attendance days and weekly hours.

Keywords: Fitness centers, customers, group exercisetraining, customer retention.

\section{Introducción}

El surgimiento de las AADD en centro deportivos puede localizarse a principios del siglo XX presentándose como una alternativa al entrenamiento cardiorrespiratorio y muscular tradicional(Conesa, 2018). Las AADD se caracterizan por realizarse en grupo mediante un instructor que enseña, guía y motiva a las personas asistentes (González-Cutre \& Sicilia, 2012; Pérez-Villalba, Baena-Arroyo, \& García-Fernández, 2017; Thompson, 2017). Existen diferentes tipos de AADD que han derivado en la definición de diversas clasificaciones en función de elementos como la intensidad de entreno, la tipología de entreno, el material utilizado o el lugar de realización de la actividad (Mata, 2015).

Según la última encuesta mundial sobre las tendencias del sector de fitness para el 2018, las AADD (en la encuesta denominadas como entrenamientos en grupo) ocupan la segunda posición en popularidad (Thompson, 2017). También se hacen eco de la demanda de este tipo de entrenamiento grupal la última encuesta de hábitos deportivos de la población espańola realizada en el año 2015 en la que un 23,9\% afirma realizar gimnasia suave y/o intensa al menos una vez a la semana (MECD, 2015). Según Veiga, Valcarce y Clavero (2018), en

Dirección para correspondencia [Correspodence address]: Marta PérezVillalba. Centro Universitario San Isidoro, Sevilla (Espańa). E-mail: marta.perez.villalba@gmail.com el estudio para la identificación de las tendencias de fitness en España para el año 2018, las clases colectivas (sinónimo de las AADD) ocuparían la posición 15 dentro del ranking de las 20 tendencias. No obstante, dentro de este ranking se encuentran tendencias de actividades que pueden ser trabajadas también a través de las AADD como el entrenamiento funcional, el entrenamiento interválico de alta intensidad (HIIT) o el entrenamiento del core. Según los autores, la tendencia de las clases colectivas "ha sido considerada como una tendencia potencial en las 11 encuestas intencionales realizadas hasta el momento por el ACSM" (Veiga et al., 2018, p. 283).

Son escasas las investigaciones, tanto a nivel internacional como a nivel espańol, que focalicen en la demanda de AADD en centros deportivos, ámbito de estudio que se correspondería al marketing de servicios deportivos (Campos, 2004). La mayoría de investigaciones llevadas a cabo no se centran en la demanda, sino en los efectos de este tipo de entrenamientos sobre la salud psicológica (González-Cutre \& Sicilia, 2012) o física (Sternlicht, Frisch, \& Sumida, 2013) de las personas, llegando a realizarse sobre el impacto de determinados sistemas de entrenamiento como Zumba ${ }^{\oplus}$ (Delextrat \& Neupert, 2016; Sternlicht et al., 2013)oBodypump ${ }^{\oplus}$ (Greco et al., 2011). Síexisten estudios que estudian la demanda global de actividades físico deportivas de la población, son las llamadas encuestas de hábitos de la población. A nivel español cada 
cinco años se realiza la encuesta de hábitos deportivos de los españoles (MECD, 2015) reproduciéndose de manera similar en la mayoría de comunidades autónomas. A pesar de que en las últimas ediciones de estas encuestas se han incorporado actividades físicas vinculadas a los centros de fitness, su tratamiento sigue siendo muy general como para conocer en profundidad el fenómeno de las AADD. Uno de los estudios realizados en España es el de Águila, Sicilia, Muyor y Orta (2009) que focaliza sobre el perfil sociodemográfico y de la práctica de los usuarios de los centros deportivos locales de una capital andaluza. Entre sus conclusiones sobre la demanda de actividades en los centros deportivos destaca la actividad de uso libre de la sala de fitness como la más demandada, especialmente entre los hombres. Respecto a las AADD, se detecta una mayor demanda por parte de las mujeres lo que podría indicar un mayor ajuste de este tipo de actividades a las motivaciones y objetivos de las mujeres. Además ellas preferirían las actividades aeróbicas con un componente musical mientras que ellos las actividades con un componente de fuerza-tonificación. Resultados similares respecto a las motivaciones de hombres y mujeres para la participación en AADD se dieron en un estudio llevado a cabo en Inglaterra (Biddle \& Bailey, 1985).

Respecto a las motivaciones en la realización de actividad física en función del género, existen estudios que asocian éstas a los estereotipos de género y a las creencias de idoneidad de una actividad en función del género. Es decir, a la supuesta existencia de actividades físicas apropiadas para los hombres pero no para las mujeres y viceversa. Un estudio llevado a cabo sobre las creencias de adecuación y capacidad de género en relación al deporte del hockey (Solmon, Lee, Belcher, Harrison, \& Wells, 2003) reveló que los hombres manifestaban una mayor seguridad en su habilidad para aprender hockey que las mujeres. No obstante, las mujeres que tenían una percepción neutra de género sobre el hockey se presentaban más seguras sobre su habilidad para aprender este deporte que las que las que lo asociaban al género masculino. Existen otros estudios en esta línea con conclusiones similares (Alvariñas-Villaverde, López-Villar, Fernández-Villarino, y Alvarez-Esteban, 2017; Hardin y Greer, 2009)que ponen de manifiesto la importancia y condicionamiento de las creencias sociales en el momento de escoger un tipo de actividad física, tanto por parte de los hombres como de las mujeres. Junto con el género, otra de las variables importantes en la demanda de servicios de actividad física y deportiva en centros deportivos es la edad de los clientes (Mullen y Whaley, 2010). El estudio llevado a cabo por la empresa Life Fitness, sobre el mercado del Fitness en España para el año 2017, señala que los "millenials" (nacidos entre 1980 y 1999) se presentan como un segmento muy importante por su gran potencial de consumo, especialmente en actividades colectivas como los entrenamientos en grupo, el HIIT y el ciclismo indoor (Life Fitness, 2017).
Dentro de la oferta de AADD también hay que hablar de la popularidad de los programas estandarizados de entrenamiento como Les Mills ${ }^{\mathrm{TM}}$ que actualmente tiene 19.500 centros deportivos con licencia para sus entrenamientos en 100 países y 130.000 entrenadores certificados (Les Mills, 2018). Estos programas buscan estandarizar las AADD, crear un marca global y comercializarlas través de franquicias y/o licenciasadaptándose al proceso de globalización actual (Parviainen, 2011). Las principales críticas hacia los programas estandarizados son el poco margen a la creatividad y adaptabilidad de los ejercicios a los usuarios que dejan a los instructores de AADD, llegándolos a comparar con un proceso de "McDonalización”. Este proceso se refiere a que el producto estándar final es efectivo, los consumidores saben el tiempo de dedicación necesario, es predecible, y por lo tanto no deja margen a sorpresas, y existe un control sobre el espacio en el que se efectúa(Andreasson \& Johansson, 2016). En el año 2013, la consultora de información Nielsen realizó un informe para Les Mills ${ }^{\mathrm{TM}}$ con una muestra de 4.610 personas abonadas a centros deportivos de 13 países. Entre los resultados del estudio destaca que la segunda motivación argumentada para estar abonado a su actual centro deportivo es la buena oferta de AADD, siendo el primer argumento la ubicación de la instalación (Nielsen, 2014a). Las AADD se presentarían como un elemento importante de la fidelización para los abonados, ya que un $46 \%$ de las personas que realizan AADD tienden a darse de baja o dejar de ir al centro deportivo si su clase preferida se cancela. Otros argumentos del informe es que el tiempo de permanencia en la instalación es superior entre las personas que hacen $\mathrm{AADD}$ y los abonados que asisten a AADD con música utilizan con más frecuencia la instalación (De La Cámara, 2015; Landa, 2009; Llamas, 2013; MorenoMurcia, Borges, Marcos, Sierra, \& Huéscar, 2012; Nielsen, 2014b).

Si bien existen diferentes trabajos que hayan manifestado las características de los clientes de centros deportivos, todavía son escasos y por tanto hay una falta de conocimiento sobre las características de las personas que asisten a estas instalaciones deportivas. Por esta razón, el objetivo del presente estudio ha sido analizar la demanda de AADD en los centros deportivos españoles, atendiendo a las variables género $y$ edadde las personas abonadas, así como valorar si las AADD funcionan como elemento de fidelización entre el colectivo de abonados.

\section{Método}

\section{Participantes}

Se estableció como población objetivo las personas abonadas a centros deportivos, de titularidad privada y pública en concesión administrativa, que ofrecen AADD y que se 
encuentran localizados en poblaciones españolas de más de 100.000 habitantes. La muestra recogida la conforman un total de 15.778 clientes de 97 centros deportivos con edades comprendidas entre los 15 y 74 ańos, de los cuales un $44,8 \%$ fueron hombres y un 55,2\% fueron mujeres. La distribución por grupos de edad se recoge en la Tabla 1.

Tabla 1. Muestra recogida según género y edad.

\begin{tabular}{llllllll}
\hline & $15-19$ & $20-24$ & $25-34$ & $35-44$ & $45-54$ & $55-64$ & $65-74$ \\
\hline Hombre & $2,6 \%$ & $8,9 \%$ & $25,2 \%$ & $30,3 \%$ & $22,6 \%$ & 10,4 & 3,7 \\
Mujer & $3,0 \%$ & $12,0 \%$ & $28,7 \%$ & $27,2 \%$ & $21,2 \%$ & $7,9 \%$ & $1,7 \%$ \\
Total & $2,8 \%$ & $10,6 \%$ & $27,2 \%$ & $28,6 \%$ & $21,8 \%$ & $9,0 \%$ & $2,6 \%$ \\
$n$ & 433 & 1.636 & 4.176 & 4.396 & 3.353 & 1.383 & 401 \\
\hline
\end{tabular}

Para la distribución entre los distintos grupos de edad se ha aplicado la utilizada en la Encuesta de Hábitos Deportivos de España (MECD, 2015).

La muestra recogida garantiza la representatividad de los resultados para el conjunto general de personas abonadas a centros deportivos espańoles. Al tratarse de un universo superior a los 100.000 casos, se ha aplicado la fórmula estadística para universos infinitos dando como resultado un error de muestra de $\pm 3 \%$ con un nivel de confianza $95 \% \mathrm{p}=\mathrm{q}=0,5$. El tipo de muestreo aplicado fue aleatorio simple probabilístico.

\section{Instrumento}

Se diseñó un cuestionario ad hoc con preguntas vinculada a la demanda de la oferta de actividad física en centros deportivos por parte de las personas abonadas. La pregunta principal, y sobre la que gira la presente investigación fue: "¿Realiza de manera regular actividades dirigidas en su centro deportivo?". Seguidamente, a las personas que respondían de manera afirmativa, se les preguntaba por la tipología de AADD que habían practicado en las últimas cuatro semanas a partir de un listado compuesto por ocho ítems que conforman las grandes tipologías de AADD en base al trabajo de Mata (2015). Con el objetivo de que las personas identificaran con mayor facilidad los diferentes grupos de AADD, éstas se acompañaban de ejemplos de actividades específicas para cada uno de los ítems tal y como se presenta a continuación:

1) Actividades coreografiadas en las que el elemento cardiovascular es el principal componente (p.ej. Zumba, Aerobic, Step, BodyCombat, Aeroboxing).

2) Actividades coreografiadas en las que el elemento tonificación-fuerza es el principal componente: (p.ej. BodyPump, CX Worx, Body Vive).

3) Actividades de tonificación-fuerza sin coreografiar (p.ej. GAP, abdominales, tonificación).

4) Actividades funcionales (p.ej. Cross-Fit, Cross Training, Cross Gym, Entrenamiento en Suspensión, TRX, HIIT).

5) Pilates Mat (suelo).
6) Actividades cuerpo-mente (p.ej. Yoga, Estiramientos, Bodyart, Body Balance, Salud Espalda, Hipopresivos).

7) Ciclismo, remo o elíptica indoor (p.ej. Spinning, Indoor Rowing, Elipdoor).

8) Actividades dirigidas en la piscina (p.ej. Aquagym, Aquafitness, Aquapilates, Aqua Cross).

9) Otras AADD.

También se pidió a las personas abonadas que indicaran el número de horas semanales dedicadas a la oferta de servicios del centro deportivo clasificando en: 1) sala de fitness; 2) AADD incluida dentro de la cuota de abono; 3) AADD no incluidas dentro de la cuota de abona (de pago extra); 4) entrenamientos personales; y 5) nado libre en la piscina.

Finalmente, se preguntó a las personas abonadas su predisposición a seguir abonadas al centro deportivo si no se ofertaran AADD.

\section{Procedimiento y análisis de datos}

La administración del cuestionario se realizó a través de una plataforma online a la que las personas encuestadas accedían mediante un enlace que el centro deportivo al que estaban abonadas les hacía llegar por correo electrónico. El trabajo de campo se realizó entre los meses de abril a julio 2017.Los datos se analizaron con el programa estadístico SPSS v.20 a través del uso de frecuencias y medias.

\section{Resultados}

Los resultados indican que un 63,3\% de las personas abonadas realizan AADD de manera regular, dándose este porcentaje más elevado en las mujeres (77,5\%) frente a los hombres $(45,9 \%)$. Atendiendo a la variable edad, los mayores índices de participación en AADD se encuentran entre los 25 y 64 años superando el $60 \%$. En el caso de las mujeres, en las franjas mencionadas, se sitúan en el 78-79\%. Los hombres entre los 15 y 19 años son los que en menor medida realizan AADD con un índice del 27,5\% (Tabla 2). 
Tabla 2. Personas que hacen AADD de manera regular según género y edad.

\begin{tabular}{|c|c|c|c|c|c|c|c|c|}
\hline & \multicolumn{8}{|c|}{ Edad en ańos } \\
\hline & $15-19$ & $20-24$ & $25-34$ & $35-44$ & $45-54$ & $55-64$ & $65-74$ & Total \\
\hline Hombre & $27,5 \%$ & $33,6 \%$ & $44,0 \%$ & $50,0 \%$ & $51,0 \%$ & $46,3 \%$ & $36,6 \%$ & $45,9 \%$ \\
\hline Mujer & $61,6 \%$ & $74,1 \%$ & $78,2 \%$ & $79,3 \%$ & $78,1 \%$ & $78,8 \%$ & $73,6 \%$ & $77,5 \%$ \\
\hline Total & $47,6 \%$ & $59,1 \%$ & $64,2 \%$ & $65,6 \%$ & $65,7 \%$ & $62,1 \%$ & $50,2 \%$ & $63,3 \%$ \\
\hline
\end{tabular}

El porcentaje de personas que hacen $\mathrm{AADD}$ de manera regular incrementa con la antigüedad de permanencia al centro deportivo. Entre las personas que llevaban menos de 3 meses abonadas al centro, el 57\% afirmaba realizar AADD de man- era regular, porcentaje que incrementa paulatinamente hasta el $69,1 \%$ de las personas con una antigüedad en el centro superior a los dos ańos (Figura 1).

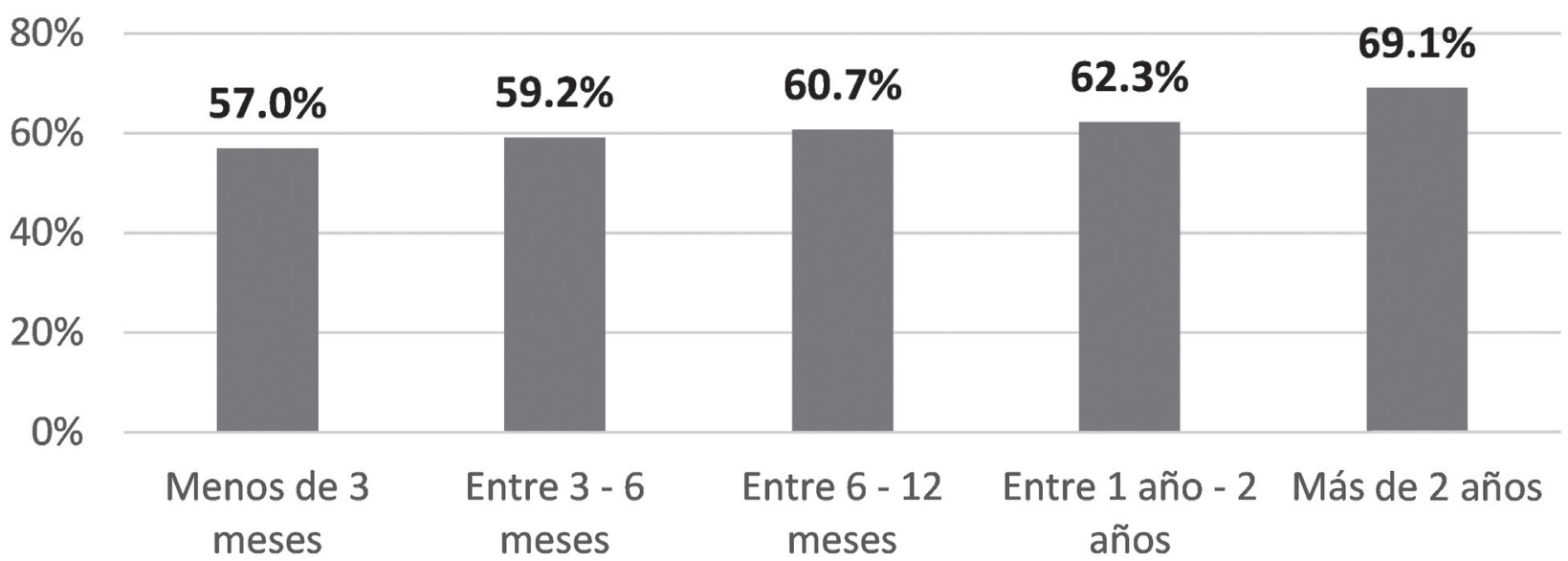

Figura 1. Personas que hacen AADD de manera regular según antigüedad.

Realizando un análisis a partir de la variable frecuencia de asistencia, se observa un mayor porcentaje de personas que hacen AADD de manera regular entre las personas que más asisten al centro con carácter semanal. En este sentido, de las personas que asisten al centro menos de una vez por semana un $21,8 \%$ hace AADD de manera regular, frente al $64,4 \%$ de la personas que tiene una frecuencia mínima de una vez por semana (Figura 2).

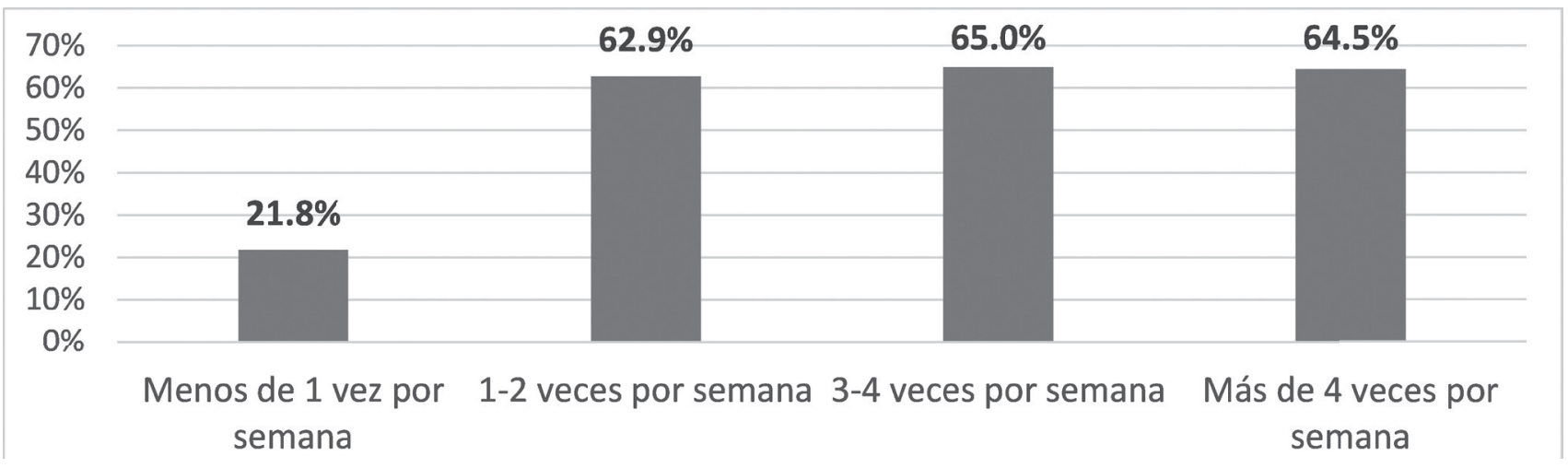

Figura 2. Personas que hacen AADD de manera regular según frecuencia semanal.

La media de horas semanales que una persona abonada a un centro deportivo invierte en la actividad física son 5,58 horas, siendo esta cuatro décimas superior en el caso de los hombres $(M=5,81)$ frente a las mujeres $(M=5,39)$ (Tabla 3). Por franjas de edad, el grupo que dedica un menor tiempo semanal es la franja de 35 a 44 años $(M=5,29)$ y el que más, la franja de los 65 a 74 años $(M$ 
$=6,80)($ Tabla 4). Dentro del tiempo invertido, la distribución de las horas se presenta de distinta manera entre hombres y mujeres. Los hombres dedican un 55,5\% del tiempo a las AADD que se traduce en una media de 3,22 horas semanales, mientras que las mujeres un 36,5\% equivalente a una media de 1,97 horas semanales. Las AADD dentro de la cuota representan el 50,4 del tiempo de las mujeres ( $M=2,72$ horas semanales) frente al $27,7 \%$ del tiempo de los hombres ( $M=1,61$ horas semanales).

Tabla 3. Distribución de las horas semanales de dedicación a la actividad física según género.

\begin{tabular}{|c|c|c|c|c|c|c|c|c|c|c|c|}
\hline & \multicolumn{2}{|c|}{ Sala de fitness } & \multicolumn{2}{|c|}{ AADD en cuota } & \multicolumn{2}{|c|}{ AADD de pago extra } & \multicolumn{2}{|c|}{ Entrenamientos personales } & \multicolumn{2}{|c|}{ Nado libre en piscina } & \multirow{2}{*}{$\begin{array}{c}\text { Total horas } \\
\text { M }\end{array}$} \\
\hline & $\mathrm{M}$ & $\%$ & $\mathrm{M}$ & $\%$ & $\mathrm{M}$ & $\%$ & $\mathrm{M}$ & $\%$ & $\mathrm{M}$ & $\%$ & \\
\hline Hombre & 3,22 & 55,5 & 1,61 & 27,7 & 0,22 & 3,7 & 0,09 & 1,5 & 0,67 & 11,6 & 5,81 \\
\hline Mujer & 1,97 & 36,5 & 2,72 & 50,4 & 0,17 & 3,1 & 0,07 & 1,3 & 0,47 & 8,8 & 5,39 \\
\hline
\end{tabular}

Nota: $\mathrm{M}=$ media de horas semanales.

La distribución de las diferentes actividades por franjas de edad también muestra diferencias. Las franjas más jóvenes (15-24 años) son las que superan el 50\% del tiempo dedicado a la sala de fitness. En cambio, a medida que avanza la edad, incrementa el interés por las AADD que se encuentran dentro de la cuota llegando a ocupar más del $40 \%$ del tiempo de realización de actividad física en las franjas de edad de 31 a 64 años (Tabla 4).

Tabla 4. Distribución de las horas semanales de dedicación a la actividad física según edad.

\begin{tabular}{|c|c|c|c|c|c|c|c|c|c|c|c|}
\hline \multirow[b]{2}{*}{ Años } & \multicolumn{2}{|c|}{ Sala de fitness } & \multicolumn{2}{|c|}{ AADD en cuota } & \multicolumn{2}{|c|}{ AADD de pago extra } & \multicolumn{2}{|c|}{ Entrenamientos personales } & \multicolumn{2}{|c|}{ Nado libre en piscina } & \multirow{2}{*}{$\begin{array}{c}\text { Total horas } \\
\mathrm{M}\end{array}$} \\
\hline & M & $\%$ & M & $\%$ & $\mathrm{M}$ & $\%$ & M & $\%$ & $\mathrm{M}$ & $\%$ & \\
\hline $15-19$ & 3,38 & 57,2 & 1,63 & 27,6 & 0,23 & 3,8 & 0,07 & 1,2 & 0,60 & 10,1 & 5,91 \\
\hline $20-24$ & 3,32 & 55,4 & 2,07 & 34,5 & 0,12 & 2,0 & 0,07 & 1,1 & 0,42 & 7,0 & 6,00 \\
\hline $25-34$ & 2,70 & 49,1 & 2,16 & 39,4 & 0,13 & 2,3 & 0,07 & 1,3 & 0,43 & 7,9 & 5,49 \\
\hline $35-44$ & 2,29 & 43,3 & 2,20 & 41,6 & 0,17 & 3,3 & 0,08 & 1,5 & 0,54 & 10,3 & 5,29 \\
\hline $45-54$ & 2,20 & 39,9 & 2,36 & 42,9 & 0,24 & 4,3 & 0,08 & 1,5 & 0,63 & 11,4 & 5,52 \\
\hline $55-64$ & 2,29 & 38,2 & 2,48 & 41,4 & 0,28 & 4,6 & 0,09 & 1,5 & 0,86 & 14,3 & 5,99 \\
\hline $65-74$ & 2,90 & 42,6 & 2,27 & 32,0 & 0,46 & 6,7 & 0,13 & 1,9 & 1,14 & 16,8 & 6,80 \\
\hline
\end{tabular}

Nota: $\mathrm{M}=$ media de horas semanales.

Entre las AADD más practicadas por los hombres destacan el "ciclismo, remo o elíptica indoor" (65,8\%), las de "tonificaciónfuerza" (31,3\%) y las "coreografiadas de tonificación” (31\%). Entre las mujeres, las "coreografiadas con componente cardiovascular" son las más populares con un $52,2 \%$, seguidas por el "ciclismo, remo o elíptica indoor" (45,4\%) y las "coreografiadas de tonificación” (39\%). Las mayores diferencias de género se dan en las "actividades coreografiadas con componente cardiovascular" llegando a ser del 32,3\% (19,9\% hombres; 52,2\% mujeres), en el "ciclismo, remo o elíptica indoor" llegando al 20,4\% (65,8\% hombres; $45,4 \%$ mujeres) y en las actividades "cuerpo-mente" llegando al 17,2\% (11,1\% hombres; $28,3 \%$ mujeres) (Tabla 5).

Tabla 5. AADD practicadas según género.

\begin{tabular}{lccc}
\hline & Hombre (\%) & Mujer (\%) & Total (\%) \\
\hline Ciclismo, remo o elíptica indoor & 65,8 & 45,4 & 52,0 \\
Coreografiadas con componente cardiovascular & 19,9 & 52,2 & 41,8 \\
Coreografiadas de tonificación & 31,0 & 39,0 & 36,4 \\
Tonificación-fuerza & 31,3 & 38,2 & 36,0 \\
Cuerpo-mente & 11,1 & 28,3 & 22,7 \\
Funcionales & 18,8 & 10,5 & 13,2 \\
Mat Pilates (suelo) & 8,4 & 22,1 & 17,6 \\
Acuáticas & 4,8 & 10,1 & 8,4 \\
Otras & 1,0 & 0,5 & 0,7 \\
\hline
\end{tabular}


Atendiendo a la variable edad, también se aprecian diferencias entre las distintas franjas (Tabla 6). La actividad más practicada en la franja de edad de los 15-19 años, las actividades "coreografiadas con componente cardiovascular", pasa del 66\% al 19,8\% en la franja de los 65-74 ańos. En el lado opuesto estarían las actividades "cuerpo-mente" que, con un
$14,6 \%$ de práctica en el colectivo de 15-19, incrementan su popularidad a medida que avanza la edad llegando al 36,1\% en la franja de 65-74 años. En el caso de las actividades funcionales, puede observarse como las franjas de mayor demanda se encuentra entre los 20-24 (17,9\%) y 25-34 (16,9\%) años.

Tabla 6. AADD practicadas según franja de edad.

\begin{tabular}{lccccccc}
\hline & $15-19(\%)$ & $20-24(\%)$ & $25-34(\%)$ & $35-44(\%)$ & $45-54(\%)$ & $55-64(\%)$ & $65-74(\%)$ \\
\hline Ciclismo, remo o elíptica indoor & 45,6 & 50,4 & 53,2 & 54,9 & 53,5 & 43,1 & 34,7 \\
Coreografiadas con componente cardiovascular & 66,0 & 53,4 & 47,4 & 40,6 & 35,5 & 31,1 & 19,8 \\
Coreografiadas de tonificación & 34,5 & 43,3 & 43,5 & 37,9 & 31,0 & 22,1 & 12,4 \\
Tonificación-fuerza & 46,5 & 49,3 & 39,9 & 33,3 & 31,4 & 28,4 & 28,2 \\
Cuerpo-mente & 14,6 & 15,1 & 20,4 & 21,0 & 26,5 & 33,3 & 36,1 \\
Funcionales & 12,6 & 17,9 & 16,9 & 13,3 & 10,2 & 6,4 & 4,0 \\
Mat Pilates (suelo) & 8,3 & 11,2 & 13,5 & 15,9 & 23,1 & 30,0 & 26,7 \\
Acuáticas & 0,7 & 3,9 & 13,2 & 22,4 & 28,2 & 22,7 & 0,7 \\
Otras & 1,0 & 0,6 & 0,7 & 0,6 & 0,6 & 0,8 & 0,5 \\
\hline
\end{tabular}

Con el objetivo de tener una visión más sintética de los datos presentados en la Tabla 5, las distintas tipologías de AADD se han agrupado en dos modalidades en la Figura 3 realizando un recalculo de los porcentajes sobre 100. El criterio de agrupación parte del nivel de exigencia: elevada exigencia cardiovascular/fuerza o media-baja exigencia cardiovascular. Las AADD de mayor exigencia pasan de una práctica del $88,5 \%$ en los 15-19 ańos a una del 50,6\% en los 65-74 ańos. Por el contrario, las AADD de menor exigencia pasan del $11,1 \%$ en los $15-19$ ańos a una del $49,1 \%$ en los 65-74 años.

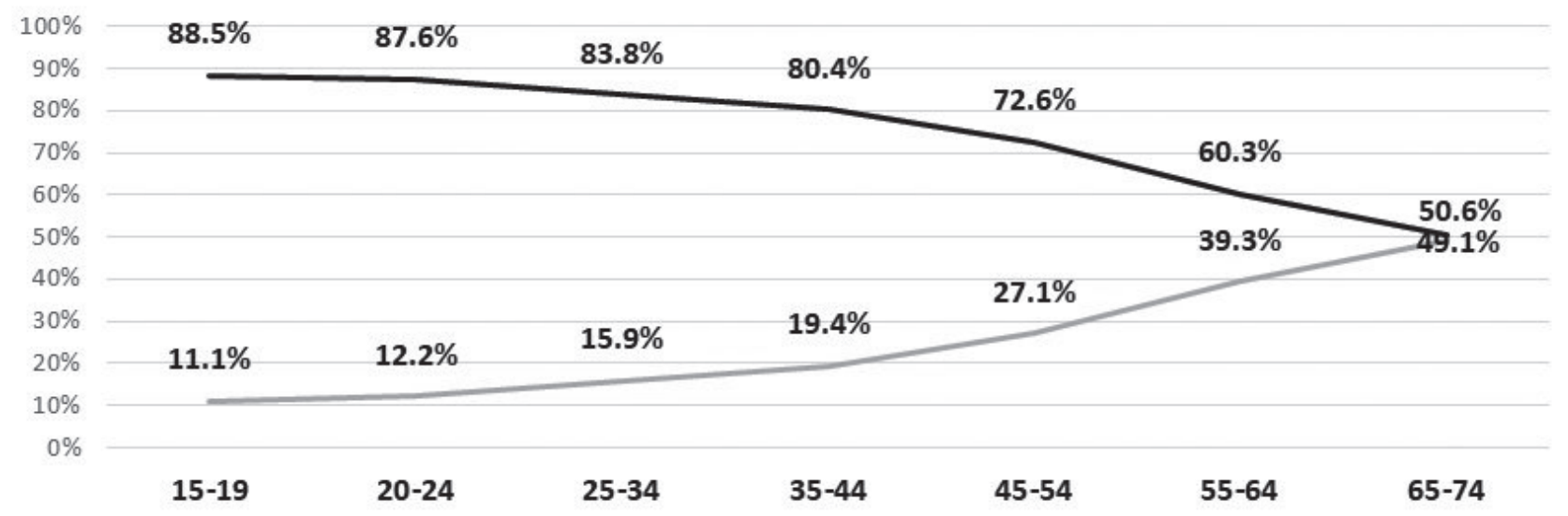

\section{—Elevada exigencia cardiovascular/fuerza —-Media-baja exigencia cardiovascular/fuerza}

Figura 3. AADD practicadas según franja de edad.

Nota: Se considera que las AADD de elevada exigencia cardiovascular/fuerza son: ciclismo indoor, coreografiadas con componente cardiovascular, coreografiadas de tonificación, tonificación-fuerza y actividades funcionales. La AADD de media-baja exigencia cardiovascular son: cuerpo-mente, acuáticas y pilates).

Por último, ante la pregunta de si la persona abonada seguiría en el centro deportivo si no se ofertaran AADD, un 58\% ha respondido que no, siendo este porcentaje más elevado entre las personas que hacen AADD de manera regular $(81,4 \%)$ frente a las que no hacen (17,7\%). Entre hombres y mujeres aparecen diferencias, un 73,2\% de las mujeres no seguirían abonadas al centro si no se ofertaran las AADD frente a un $39,4 \%$ de los hombres. Hay que tener presente que estos resultados están vinculados a los porcentajes de práctica de AADD entre hombres y mujeres tal y como también sucede 
al analizar los datos por franjas de edad (Figura 4). Dentro del colectivo de personas que hacen AADD de manera regular, la diferencia de porcentajes entre la práctica regular y el hecho de no seguir abonado si no se ofertaran AADD es del $3,7 \%$ en la franja de 25 a 34 ańos y del $12,7 \%$ en la de 65 a 74 ańos.

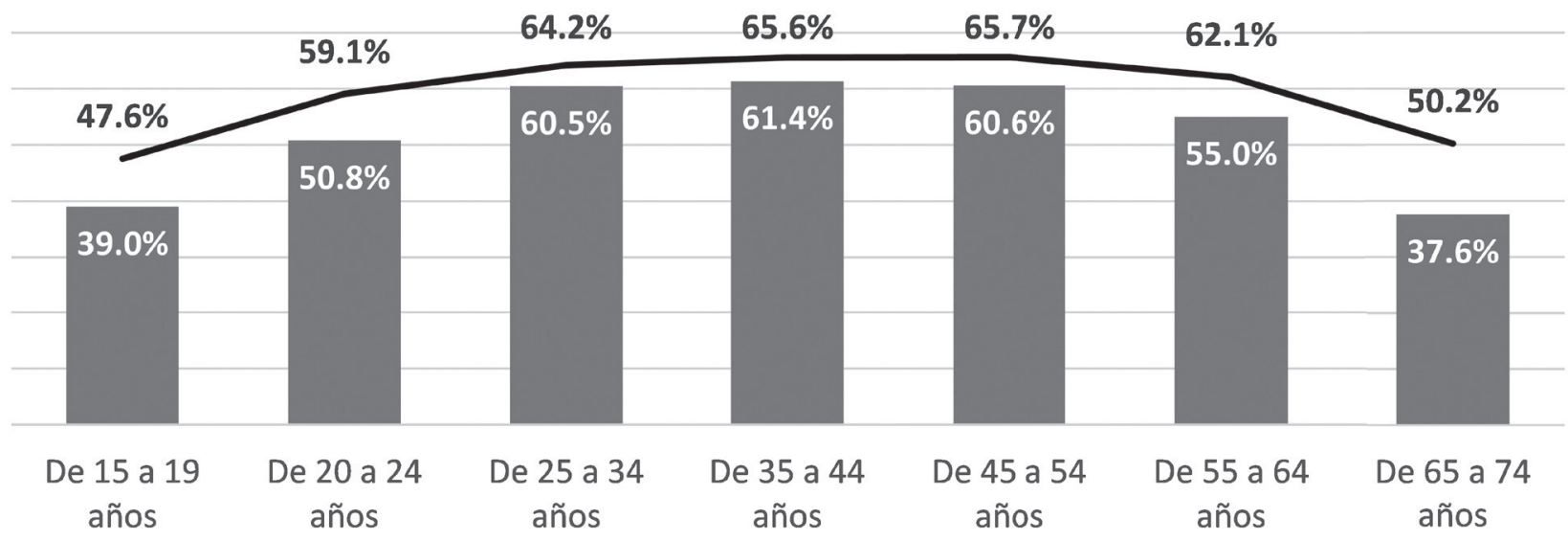

Personas que no seguirían abonadas

_-Personas que hacen AADD de manera regular

Figura 4. Personas que no seguirían abonadas a su centro deportivo si no se ofertaran AADD.

\section{Discusión y conclusiones}

Los resultados de la investigación ponen de manifiesto la importancia de las variables género y edad en la demanda de las AADD. Los datos muestran coincidencias con los resultados del estudio llevado a cabo por Águila et al. (2009), donde los hombres invierten más tiempo en la sala de fitness y las mujeres en la realización de AADD. Estas diferencias también se producen en el tipo de AADD practicada: las más populares entre las mujeres son las coreografiadas con componente cardiovascular y el ciclismo indoor, coincidiendo con el gusto por las actividades coreografiadas y con soporte musical detectado en el estudio de Biddle y Bailey (1985). Para los hombres, el ciclismo indoor se presenta como la principal actividad seguida por las de tonificación-fuerza. Por lo tanto, el ciclismo indoor, como AADD, parece responder tanto a los intereses/motivaciones de práctica de mujeres y hombres. El desequilibrio entre géneros en la práctica de las diferentes modalidades de AADD podría explicarse por la creencia de que existen actividades que serían más adecuadas para los hombres que para las mujeres y viceversa. Esta creencia se daría como consecuencia de la creación de estereotipos de género durante el proceso de socialización de las personas (Alvariñas-Villaverde et al., 2017; Hardin y Greer, 2009; Solmon et al., 2003).

El análisis por franja de edad muestra una evolución de la demanda de AADD a medida que incrementa la edad de las personas usuarias. A grande rasgos, se podría decir que entre las franjas más jóvenes existe una mayor dedicación del tiempo a la sala de fitness que va desapareciendo en favor de las AADD a medida que incrementan los ańos. Se desconoce si esta ten- dencia tiene que ver con una evolución de los intereses a medida que avanza la edad o bien, con cambios generacionales en los patrones de motivaciones a la hora de hacer actividad física que se mantendrán estables a lo largo de los años. Profundizando en la tipología de AADD practicada, los más jóvenes prefieren aquellas que tienen una elevada exigencia cardiovascular y/o de fuerza frente a los más mayores que se decantan por actividades de exigencia media-baja. Por ejemplo, en el caso de las actividades funcionales (p.ej. Cross-Fit, Entrenamiento enSuspensión, HIIT) se ha podido observar como las franjas de edad correspondientes a los "millenials" (20-24 y 25-34 años) son las que más demandan este tipo de actividades tal y como constaba el estudio de Life Fitness (2017).

Los datos que arrojan el estudio refrendan la hipótesis planteada de que las AADD funcionarían como un elemento de fidelización de las personas abonadas, pues tal y como se ha podido corroborar, las personas que realizan de manera regular AADD presentan una mayor antigüedad como abonado, una mayor frecuencia semanal de asistencia al centro, así como un mayor volumen de horas. Estos datos coinciden con el estudio llevado a cabo por Nielsen(2014a, 2014b) para la empresa de programas de entrenamiento grupal Les Mill$s^{\mathrm{TM}}$. Además, ante la pregunta de si la persona seguiría acudiendo a la instalación si no se ofertaran AADD, más de tres partes $(81,4 \%)$ de las personas que hacen AADD de manera regular ha respondido negativamente.

La escasez en la literatura, tanto internacional como nacional, sobre la temática de estudio se ha presentado como una debilidad tanto a la hora de definir el objeto teórico, el instrumento, así como en la interpretación de los resultados. Por ello, 
una de las limitaciones del estudio es el carácter sincrónico de la investigación que no permite concluir si las diferencias entre franjas de edad se deben cambios en las pautas de comportamiento de los usuarios a través de los años o bien, a cambios generacionales. El diseño de un cuestionario ad hoc para esta investigación hay que señalarlo como un elemento importante a tener en cuenta en la interpretación de los resultados y las conclusiones que se derivan del presente artículo. Por último, otras de las limitaciones tienen que ver con la clasificación realizada de las AADD, pues el hecho de agruparlas de una determinada manera puede haber influido en los resultados obtenidos.

Como posibles líneas de futuro, se requiere de estudios que analicen en profundidad el impacto de la variable edad en la demanda de AADD desde una perspectiva longitudinal. Igualmente, se propone en futuras investigaciones que se también se analice el comportamiento del cliente en centros deportivos públicos gestionados de manera directa, ya que este estudio se ha centrado en aquellos titularidad y/o gestión privada. Además, sería interesante que el cuestionario diseñado para esta investigación pueda ser aplicado en otros estudios con el objetivo de garantizar su validez.

Igualmente, los hallazgos derivados de este estudio ayudan a los directores de los centros deportivos a su gestión diaria. De hecho, las implicaciones para la gestión son muy claras. Por un lado, conocer la demanda de los abonados segmentada por las variable sociodemográficas de género y edad les permite poder realizar una mejor planificación técnica de las actividades a ofertar en sus centros. Por el otro, el hecho de saber que las AADD funcionan como elemento de fidelización y retención proporciona una información valiosa para la reorientación de las política de fidelización y retención de clientes, tanto de los que demandan AADD como de los que demandan otro tipos de servicios deportivos como puede ser la sala de fitness.

\section{Anexo}

A continuación se detallan las preguntas del cuestionario diseñado y utilizado en la investigación:
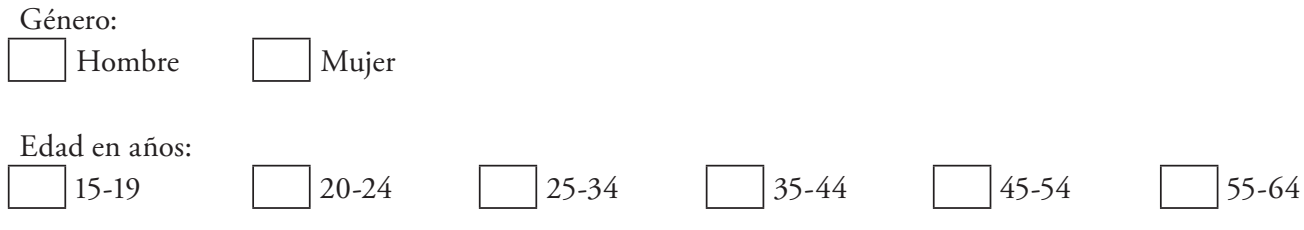
$65-74$

¿Cuánto tiempo lleva inscrito/a a su centro deportivo?

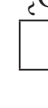
Menos de 3 meses

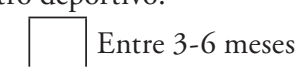

Más de 2 años

¿Con qué frecuencia semanal asiste al centro deportivo?

Menos de 1 vez por semana

$\square$ veces por semana

\section{$\square$ vez por semana} 4 veces por semana
2 veces por semana

Más de 4 veces por semana

Indique el número de horas semanales que dedica a lassiguientes actividades:

$\square$ Sala de fitness

Actividades dirigidas (en sala y/o piscina) incluidas en la cuota

Actividades dirigidas (en sala y/o piscina) NO incluidas en la cuota (de pago extra)

Entrenamiento personal (de pago extra)

Nado libre en piscina 
¿Realiza de manera regular actividades dirigidas en su centro deportivo?

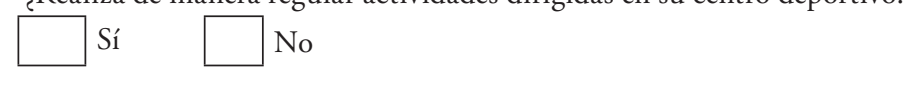

Indique el tipo de actividades dirigidas que ha realizado en las últimas 4 semanas

Actividades coreografiadas en las que el elemento cardiovascular es el principalcomponente (p.ej. Zumba, Aerobic, Step, BodyCombat, Aeroboxing).

Actividades coreografiadas en las que el elemento tonificación-fuerza es el principalcomponente: (p.ej. BodyPump, CX Worx, Body Vive).

Actividades de tonificación-fuerza sin coreografiar (p.ej. GAP, abdominales, tonificación).

Actividadesfuncionales (p.ej. Cross-Fit, Cross Training, Cross Gym, Entrenamiento en Suspensión, TRX, HIIT).

Pilate Mat (suelo).

Actividades cuerpo-mente (p.ej. Yoga, Estiramientos, Bodyart, Body Balance, SaludEspalda, Hipopresivos).

Ciclismo, remo o elíptica indoor (p.ej. Spinning, Indoor Rowing, Elipdoor)

Actividades dirigidas en la piscina (p.ej. Aquagym, Aquafitness, Aquapilates, AquaCross)

Otras

Si su centro deportivo no ofertara actividades dirigidas, ¿seguiría abonado/a?

$\square$ Sí $\square$ No

\section{Agradecimientos}

Este proyecto ha sido financiado por Aerobic \& Fitness S.L.

\section{Referencias}

1. Águila, C., Sicilia, A., Muyor, J. ., y Orta, A. (2009). Cultura posmoderna y perfiles de práctica en los centros deportivos municipales. Revista Internacional de Medicina y Ciencias de La Actividad Física y El Deporte, 9(33), 81-95.

2. Alvarińas-Villaverde, M., López-Villar, C., Fernández-Villarino, M. A., y Alvarez-Esteban, R. (2017). Masculine, feminine and neutral sports: Extracurricular sport modalities in practice. Journal of $\mathrm{Hu}$ man Sport and Exercise, 12(4), 1278-1288. http://doi.org/10.14198/ jhse.2017.124.14

3. Andreasson, J., y Johansson, T. (2016). 'Doing for group exercise what McDonald's did for hamburgers': Les Mills, and the fitness professional as global traveller. Sport, Education and Society, 21(2), 148-165. http:// doi.org/10.1080/13573322.2014.885432

4. Biddle, S., y Bailey, C. (1985). Motives for participation and attitude toward physical activity of adult participants in fitness programs. Perceptual and Motor Skills, 61, 831-834. http://doi.org/doi/abs/10.2466/ pms.1985.61.3.831

5. Campos, C. (2004). Dirección y marketing de servicios deportivos. Barcelona: GPE.
6. Conesa, E. (2018). Intensidad del entrenamiento personal dirigido en modalidades de danza y baile. SPORT TK: Revista Euroamericana de Ciencias Del Deporte, 7(1), 123-128.

7. De La Cámara, A. (2015). El sector del fitness en España; análisis del gimnasio low-cost y los centros de electroestimulación integral. SPORT TK-Revista EuroAmericana de Ciencias Del Deporte, 4(2), 47-54.

8. Delextrat, A., y Neupert, E. (2016). Physiological load associated with a Zumba ${ }^{\circledR}$ fitness workout: A comparison pilot study between classes and a DVD. Journal of Sports Sciences, 34(1), 47-55. http://doi.org/10.1080/ 02640414.2015 .1031162

9. González-Cutre, D., y Sicilia, A. (2012). Dependencia del ejercicio físico en usuarios españoles de centros de acondicionamiento físico (fitness): Diferencias según el sexo, la edad y las actividades practicadas. Behavioral Psychology, 20(2), 349-364.

10. Greco, C., Oliveira, A., Pereira, M., Figueira, T., Ruas, V., Gonçalves, M., y Denadai, B. (2011). Improvements in metabolic and neuromuscular fitness after 12-week Bodypump $(\mathrm{R})$ training. Journal of Strength and Conditioning Research, 25(12), 3422-3431. 
11. Hardin, M., y Greer, J. D. (2009). The Influence of Gender-role Socialization, Media Use and Sports Participation on Perceptions of GenderAppropriate Sports. Journal of Sport Behavior, 32(2), 207-226.

12. Landa, M. I. (2009). Subjetividades y consumos corporales: un análisis de las prácticas del fitness en España y Argentina. Razón y Palabra, 14(69).

13. Life Fitness. (2017). El mercado del fitness en España. Barcelona. Descargado desde https://www.lifefitness.es/sites/g/files/dtv376/f/Zoom Mercado 2017_LifeFitness.pdf

14. Llamas, J. C. (2013). Determinación de prácticas saludables en programas de coreografías de fitness y aeróbic: dance dance revolution, Les Mills y Base Training. European Journal of Human Movement, 30, 85-102.

15. Mata, E. (2015). Avances en clases colectivas: nuevas disciplinas. In B. Sañudo \& J. García-Fernández (Eds.), Nuevas orientaciones para una actividad fisica saludable en centros de fitness (pp. 147-166). Sevilla: Wanceulen.

16. MECD. (2015). Encuesta de hábitos deportivos en España 2015. Madrid. Descargado desde https://www.mecd.gob.es/servicios-al-ciudadano$\mathrm{mecd} / \mathrm{en} / \mathrm{dms} / \mathrm{mecd} /$ servicios-al-ciudadano-mecd/estadisticas/deporte/ ehd/Encuesta_de_Habitos_Deportivos_2015.pdf

17. Moreno-Murcia, J. A., Borges, F., Marcos, P., Sierra, A. C., y Huéscar, E. (2012). Motivación, frecuecia y tipo de actividad en practicantes de ejercicio físico. Revista Internacional de Medicina y Ciencias de La Actividad Física y Del Deporte, 12(48), 649-662.

18. Mullen, S. P., y Whaley, D. E. (2010). Age, gender, and fitness club membership: Factors related to initial involvement and sustained participation. International Journal of Sport and Exercise Psychology, 8(1), 24-35. http://doi.org/10.1080/1612197X.2010.9671931

19. Nielsen. (2014a). Consumer fitness trends: Statistics \& insights for fitnes facilities. Descargado desde https://w2.lesmills.com/files/GlobalCentral/GRIT/ResearchDocs/Nielsen research 2013 exec summary.pdf

20. Nielsen. (2014b). Les Mills Global Consumer Fitness Survey (2014).

21. Parviainen, J. (2011). The standardization process of movement in the fitness industry: The experience design of les mills choreographies. European Journal of Cultural Studies, 14(5), 526-541. http://doi. org/10.1177/1367549411412202

22. Pérez-Villalba, M., Baena-Arroyo, J., y García-Fernández, J. (2017) Actividades dirigidas virtuales: Un análisis de la oferta y la demanda en centros deportivos. Journal of Sports Economics \& Management, 7(2), 101-110.

23. Solmon, M. A., Lee, A. M., Belcher, D., Harrison, L., y Wells, L. (2003). Beliefs about Gender Appropriateness, Ability, and Competence in Physical Activity. Journal of Teaching in Physical Education, 22(3), 261279. http://doi.org/10.1123/jtpe.22.3.261

24. Sternlicht, E., Frisch, F., y Sumida, K. D. (2013). Zumba Fitness work outs: Are they an appropriate alternative to running or cycling? Sport Sciences for Health, 9(3), 155-159. http://doi.org/10.1007/s11332-0130155-8

25. Thompson, W. R. (2017). Worldwide survey of fitness trends for 2018: The CREP Edition. ACSM's Health and Fitness Journal, 21(6), 10-19. http://doi.org/10.1249/FIT.0000000000000341 